This item was submitted to Loughborough's Research Repository by the author.

Items in Figshare are protected by copyright, with all rights reserved, unless otherwise indicated.

\title{
Innovative mechanism to identify robot alignment in an automation system
}

PLEASE CITE THE PUBLISHED VERSION

https://doi.org/10.1016/j.robot.2019.01.015

PUBLISHER

(c) Elsevier

VERSION

AM (Accepted Manuscript)

PUBLISHER STATEMENT

This paper was accepted for publication in the journal Robotics and Autonomous Systems and the definitive published version is available at https://doi.org/10.1016/j.robot.2019.01.015

\section{LICENCE}

CC BY-NC-ND 4.0

\section{REPOSITORY RECORD}

Millington, Joseph, Radmehr Monfared, and Daniel A. Vera. 2019. "Innovative Mechanism to Identify Robot Alignment in an Automation System". Loughborough University. https://hdl.handle.net/2134/36722. 


\title{
Innovative Mechanism to Identify Robot Alignment in an Automation System
}

\author{
Joseph Millington ${ }^{a}$, Radmehr P Monfared ${ }^{b}{ }^{*}$, Daniel Vera ${ }^{c}$ \\ a Gazprom Energy, UK \\ ${ }^{b}$ Mechanical, Electrical, and Manufacturing Engineering Department, Loughborough University, UK \\ c Automation Systems Group, WMG - International Manufacturing Centre, University of Warwick, UK
}

\begin{abstract}
Robotic applications are commonly used in industrial automation systems. Such systems are often comprised of a series of equipment, including robotic arms, conveyors, a workspace, and fixtures. While each piece of equipment may be calibrated with the highest precision, their alignment in relation to each other is an important issue in defining the accuracy of the system. Currently, a variety of complex automated and manual methods are used to align a robotic arm to a workspace. These methods often use either expensive equipment or are slow and skill-dependent.

This paper presents a novel low-cost method for aligning an industrial robot to its workcell at 6 degrees of freedom (DoF). The solution is new, simple and easy to use and intended for the SMEs dealing with low volume, high complexity automated systems. The proposed method uses three dial indicators mounted to a robot end effector and a fixed measurement cube, positioned on a workcell. The robot is pre-programmed for a procedure around the cube. The changes on the dial indicators are used to calculate the misalignment between the robot and the workcell. Despite simplicity of the design, the solution is supported with complex real-time mathematical calculations and proven to identify and eliminate misalignment up to $3 \mathrm{~mm}$ and 5 degrees to an accuracy of $0.003 \mathrm{~mm}$ and 0.002 degrees: much higher than the precision required for a conventional industrial robot.
\end{abstract}

In this article, the authors describe a proposed solution, validate the computation both theoretically and through a laboratory test rig and simulation.

Keywords: Robot alignment, Automated systems, Robot calibration, Forward and Inverse modelling

\section{Introduction}

In recent years, robot calibration and alignment has become an increasingly important issue in the field of industrial robots. Robotic field of application has broadened significantly due to a growing number of robot assisted tasks performed in industry today. The use of robot manipulators has changed many industries such as the automotive industry, increasing the cost effectiveness and efficiency of production lines.

In the majority of industrial applications, the programming of a robot manipulator is done using manual Teach-In programming, or off-line programming. The programming methods have advanced significantly from simply using pendent, to the current offline methods [1]. Nonetheless, it is a tedious and slow process and relies heavily on the operator's skills, with the level of accuracy varying between different operators. However, along with the evolution of 3D CAD and solid-modelling software, the advent of off-line programming methods has since eliminated many of the issues with manual programming method with, in many cases, time-savings of up to 85\% [2], [3].

Regardless of the method of programming used, the lack of accuracy in the geometrical positioning of a robot manipulator in relation to the other components of the automation system such as

\footnotetext{
${ }^{*}$ Corresponding author - R.P.Monfared@lboro.ac.uk ORCID 0000-0002-8782-3426
} 
workpieces, conveyors, fixtures and other manipulators is a critical step in configuring a robotic system. This is where robot alignment techniques become necessary.

Traditionally, robots are calibrated based on manufacturers' instructions and special purpose devices. But the accurate co-ordination of a robot in relation with a system is typically measured manually, using pointed objects and skilled labours, or using measurement devices such as laser trackers [4]. Clearly this configuration measurement has profound impact on the final precision of the system.

The manual alignment method is typically low-cost with a skill-dependent level of accuracy and is suitable for a limited number of robots. On the other hand, precision measurement devices offer ultimate accuracy; but could be highly expensive, are appropriate for only large numbers of alignment processes and at a very high cost.

In this article, the authors report design and development of a low-cost alignment method of a sufficient precision to fully align industrial robotic arms' reference coordinate with a workspace in an automation system. The article does not discuss the standard robot calibration processes as we assume that the robots used in our alignment procedure are already calibrated using a standard manufacturer-recommended method. The proposed mechanism is designed for SMEs in mind with a limited number of robots, which perform high accuracy tasks. Therefore, the maximum cost of the proposed automated solution is considered to be comparable with the annual cost for the manual alignment operations, estimated to be around $£ 1000$.

The novelty for the unique proposed solution includes ease and speed of use, a capability to be integrated into the robot control system (hence semi to full automated), high precision alignment with a very low investment cost. The solution provides alignment in 6 DOF within a range of $30 \mu \mathrm{m}$, for a robot that is misaligned up to $3 \mathrm{~mm}$ in translation, and $5^{\circ}$ in rotation.

\section{Prior knowledge}

At present, most techniques used to perform robot alignment process require human interaction for each individual robot, or have to be done under laboratory conditions through a long and cost intensive process. In the former case, the human tasked with aligning the robot has to align the end effector of the manipulator to the workpiece using the eyes. Clearly this is time/human-power consuming, unreliable, and inefficient. In the latter case, expensive and highly complicated measurement systems, such as tracking laser interferometers, which have to be operated by highly trained personnel, align manipulators to a far higher degree of accuracy than is necessary for the majority of robotic applications. Furthermore, current alignment methods necessitate robot arm motion. This joint movement introduces additional alignment inaccuracies due to the limited robot repeatability, reducing the overall accuracy of the alignment process.

Arguably the most basic and primitive method of aligning a manipulator to a workpiece or work surface is to manually measure the position of the tool centre point by means of a dial gauge. By calculating its displacement from the desired position along three perpendicular axes, the translational misalignment can be corrected by inputting the adjustments necessary to re-align the manipulator into the robot controller. Apart from the fact that this is unreliable and time consuming, this method typically only determines the positional misalignment of the robot, and not the rotational misalignment that might be present due to an angular offset at the robot base. The rotational alignment may be essential for certain automated processes such as robotic welding.

The theoretical aspect of the robot calibration process has been studied since 1990s by various research groups, including Kurzon [5], Kukareko [6], and later on by Zhenhua [7]. A number of experimental and commercial equipment are available to define the true home position of a robot, such as Renishaw telescoping Ballbar [8] and other methods using various artefacts such as an Open/Partial-cube [9] located within the robot workspace. Typically strain-gauged used to measure the 
contact point of the robot with the artefacts. These methods facilitate calibration of the robot at its home position by comparing the actual position with the programmed co-ordinations.

One common approach regarding the alignment of industrial robots is the use of theodolite system[10]-[12]. Theodolite triangulation systems provide real-time 3D coordinates of target points located on the manipulator's end effector. Automatic tracking, focusing, and centring provide the alignment system with speed, reliability, and repeatability [10], [13]. Only static 3DOF measurements can be acquired, but the devices are portable and do not require direct contact with the robot arm. Attainable accuracy is dependent on numerous factors, especially geometrical configuration, but Leica [14], a leading supplier of theodolite systems, claims it can be up to $10 \mu \mathrm{m}$.

Driels and Pathre [13] demonstrate an automatic theodolite system used to align a six axis PUMA 560 robot to a cubical work piece in the laboratory. The results of this experiment show that after 100 observations of a target fixture, automatic theodolite alignment methods can reduce the inaccuracies caused by the installation misalignment by an order of magnitude. Kyle [10], [15] demonstrates the basic principle for determining the 3D location of any target point of interest by triangulation. However, it is mentioned that before the points of interest can be located by the theodolites, the relative position and angular attitude of the theodolites must be determined. This lengthy technical set up limits their applicability on the shop floor due to the possible lack of expertise. As well as this constraint, wide scale application of theodolite systems is hindered by the cost, which is of the order of $f 50,000$ [14], which is unlikely to be within the budget of many small scale businesses.

Leica and many other companies offer alternative solutions to the robot alignment problem in the form of tracking laser interferometers [16], [17]. Like theodolite triangulation systems, the most basic tracking laser interferometers have been developed for obtaining 3D positional information only. A number of retroreflectors are mounted on the manipulator end effector and automatically tracked by a moving laser beam. They are amid the most accurate alignment systems, and incorporate the advantages of very high precision, contactless measurements, and portability. Again, similar to the theodolite method, the most basic form of laser tracker requires at least three target retroreflectors to be able to indirectly determine the 6 DOF at the end effector.

However, there exist more sophisticated systems which have the ability to measure both position and orientation directly. The measurement principle for such a system utilizes a CCD-camera [18] to analyse the intensity profile of a laser beam, reflected from a single retroreflector mounted on the robot's end effector. From this intensity profile analysis, as explained by Prenninger [19], one can uniquely determine the orientation of the robot's end effector, thus directly determining the 6 DOF to completely align the robot. Nowadays, the accuracy of such laser systems can be up to $8 \mu m$ [20], which is higher than what is required for most robot manipulators, which have a typical repeatability of $0.05 \mathrm{~mm}$ [21]. This high degree of accuracy however comes at a considerable cost. For example, including software training, the FARO Vantage Laser Tracker may cost up to $£ 85,000$.

A slightly less common method of robot alignment involves the use of one or more camera sensors either fixed to the robot end effector or positioned in the proximity of the robot. In the former case, a single camera may be used to observe reference points on a reference object, enabling the relative position of the object to be reconstructed using space resection techniques [22]. In the latter case, two or more stationary cameras may be used to observe a target mounted on the end effector to reconstruct the robot position and orientation using stereo vision. However, the accuracy of this approach is dependent on the quality of the device (at considerable cost) and the environment light.

In addition, in a similar research domain, Kalt[23] and Du [24] have developed methods to facilitate interface between human motions and robotic control system using a variety of sensor fusions. While these researches are not completely relevant to this article, the computational approaches proposed by them are found to be in line with the methods proposed in this article.

From this review, it can be seen that a large number of robot alignment systems are commercially available, each with its own range of applicability and its own requirements. Prices and required levels of expertise vary over a broad spectrum. However, there is a gap in the market for a rapid, affordable, 
and adequately accurate alignment system to suit the needs of small to medium automated operations, as illustrated by Figure 1. The proposed alignment solution is envisaged to support the small businesses for the targeted gap in the market. The cost of the proposed solution is estimated to be between $£ 100$ to $£ 300$, depending on the quality of the measurement devices.

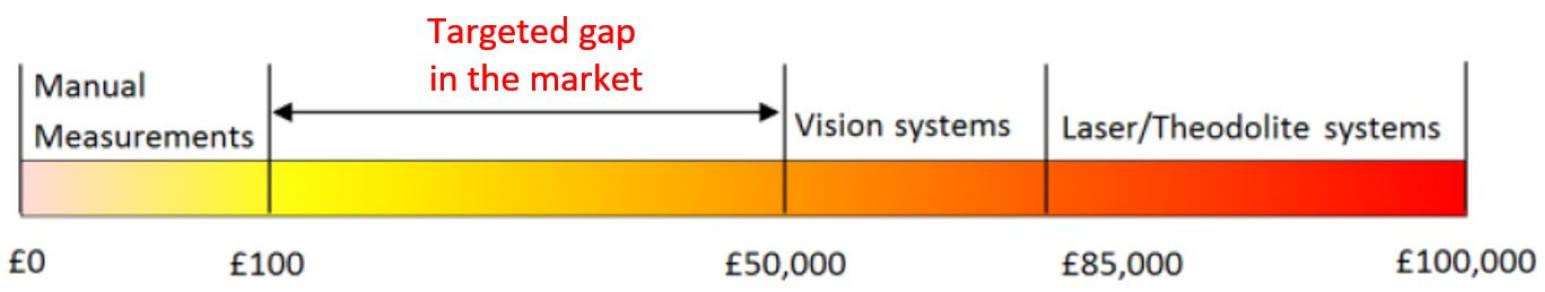

Figure 1: Current solutions to robot alignment are either manual, inaccurate and inefficient, or far too expensive with unnecessarily high accuracy.

\section{Alignment in automation systems}

An automation system may consist of many components including robotic arms, measurement devices, conveyors, vision systems, and movable tables. While each component may have a high level of precision in respect to their own static dimensions and dynamic motions, the geometrical relationship between the components will have a critical role in the accuracy of the overall automation system. Therefore, the alignment concept discussed here is at a system level as a whole and not at device level. Nonetheless, the article is focused on the alignment of robotic arms in relation with the other components such as a workspace.

Theoretically, the position and orientation of a body in space can be obtained by attaching a coordinate system (or frame) rigidly to the body, and then describing the position and orientation of that frame with respect to a reference coordinate system. A typical robot controller defines four right handed Cartesian coordinate systems [25][26] as illustrated by Figure 2.
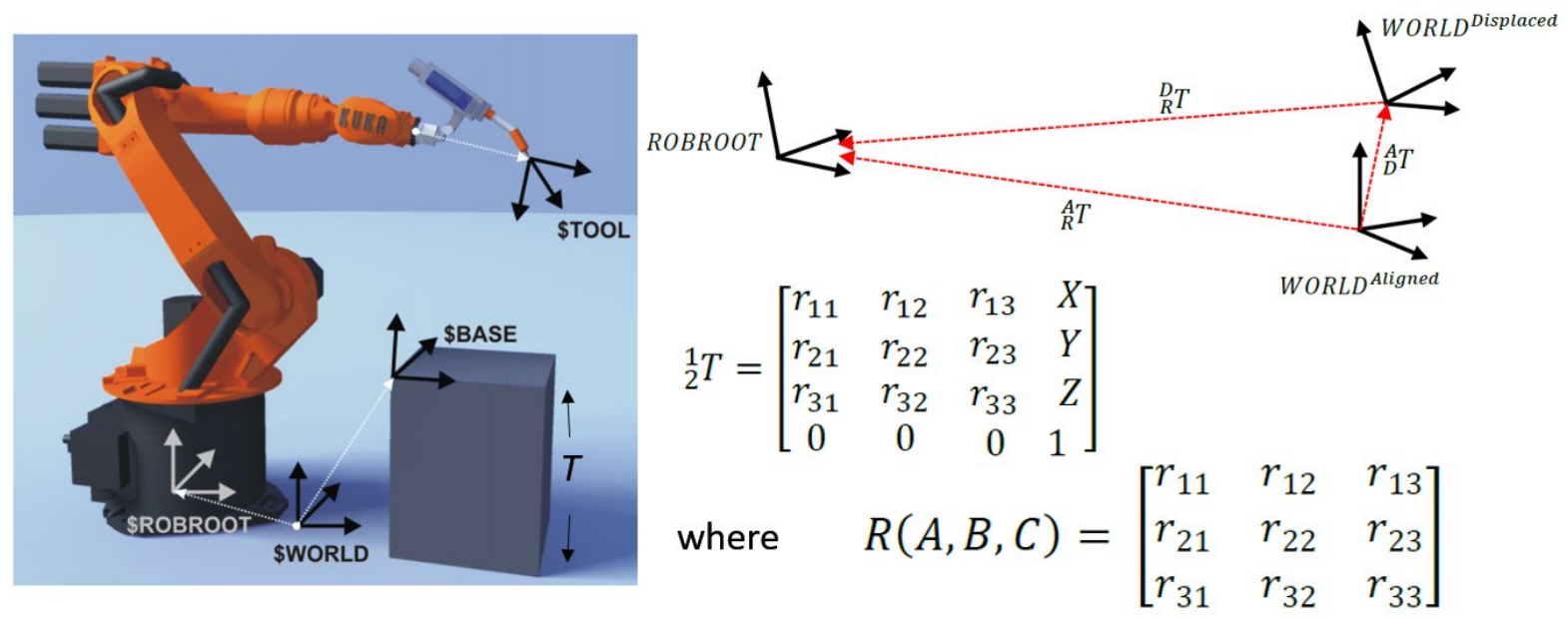

Figure 2: An overview of the robot coordinate systems

The ROBROOT coordinate system is located at the base of the robot and describes the relative position and orientation of the robot. If the translations in positive direction are $x, y, z$ and rotations around the three axes are $A, B, C$ using conventional homogenous transform matrixes any point from one frame (e.g. a displaced coordination) can be mapped to the other frame (e.g. a fully aligned coordination), as shown by Figure 2. Accordingly, three matrixes can describe the relationships 
between the ROBROOT, initial WORLD (i.e. perfect aligned system) and actual WORLD (i.e. misaligned or displaced) coordinate systems, namely ${ }_{R}^{D} T,{ }_{R}^{A} T$ and ${ }_{D}^{A} T$.

${ }_{D}^{A} T \in R^{4 \times 4}$ denotes the homogeneous transformation matrix mapping the initial (aligned) WORLD frame to the actual displaced WORLD frame. It can then be said that the target problem is to solve the homogenous transform ${ }_{R}^{D} T$ in order to extract the real $x, y, z, A, B, C$ values that enable the robot controller to redefine the offset between the ROBROOT coordinate system and actual WORLD coordinate system (i.e. misalignment between the two systems). The offset values can be then automatically fed into the robot controller to accommodate such unavoidable geometrical misalignments between the robot and a system.

\subsection{Proposed alignment solution}

A number of high precision and high cost methods of alignment were reviewed and it was concluded that the majority of the smaller users (e.g. SMEs) require a low cost semi-automated method with a sufficient precision level.

The novel 6 DoF robotic alignment method proposed by the authors is illustrated by Figure 3 . The proposed method consists of a measurement disk comprising three dial indicators and a calibration cube. The measurement disk with a defined dimensions would be mounted on a robot arm at a coordination pre-programmed and known to the robot. The calibration cube will be placed and fixed on a workspace, such as a table or a fixture, where the robot has to be aligned to. The calibration cube would have surface quality in range of 0.8 to $1 \mu \mathrm{m}$ roughness average to minimise the interference with the measurement process.

Assuming initially that the robot is in full alignment with the workspace, the robot is programmed to move to the theoretical position at the corner of the cube, where the centre of the robot $6^{\text {th }}$ axis passes through the corner and the centre of the cube (i.e. vector $V$ in the figure) and the dial indicators touch the cube surface from three directions. The measurement disk then will rotate slightly to the left and then to the right by the robot to generate 6 values produced by the contraction or extension of the dial indicators. A 2-degree rotation was found practical for this experiment. This is due to a number of variables. Theoretically, any small rotation value should be adequate to complete the calculations. However, the rotation value is also limited with the physical constraints. These include the minimum value measureable with the dial indicators, the surface quality of the cube, and the increase in the risk of deflection of the measurement probes at larger rotation values.

Those observed 6 values from the indicators will then be used to specify the misalignment between the robot and the calibration cube (or the workspace) at six degree of freedom. Clearly, in a perfect aligned situation, the 2 sets of extracted values from the indicators should be identical. However, in a misaligned or displaced system, the difference between the 2 sets of values can theoretically determine the misalignments at 6 degrees of freedom, within an acceptable margin of error for the targeted application domain.

The novelty of the proposed approach is in its simplicity in physical design, which is supported by complex real-time mathematical calculations, embedded into the physical system. The approximation methods used in the proposed method enables instantaneous calculation of the misalignment values by maintaining a level of mathematical accuracy compatible with the physical precision of an industrial robot. Using digital indicators (with automatic data reading mechanism), the system can be integrated to a robot controller to rapidly offset the robot reference coordination based on the calculated values at real-time. The process could be repeated until the calculated misalignment between the two systems reduces to an acceptable threshold. 

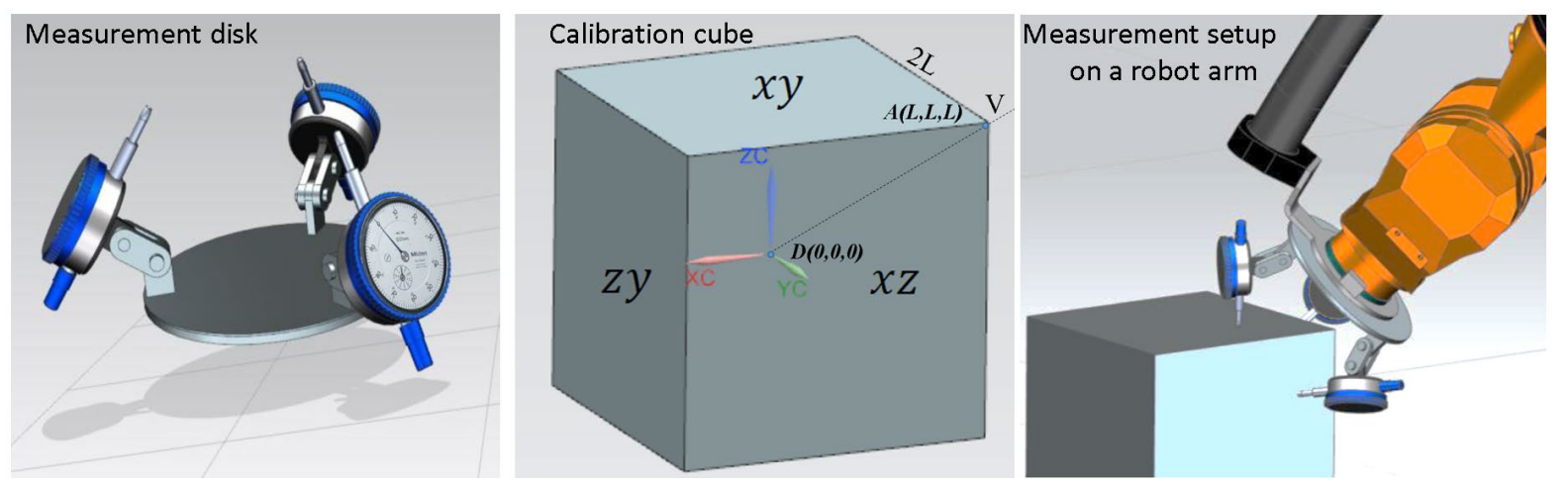

Figure 3: Proposed semi-automated robotic alignment method

Initially, an algorithm was developed to solve the forward problem of computing the coordinates of the indicators' contact points with the cube surface at both perfectly aligned position and a known displaced position. This is used to verify the following algorithm that will actually be used during the alignment process.

This algorithm calculates coordination of the contact points for both $\phi$ and $-2 \phi$ degrees rotation of the measurement disk. The accuracy of the algorithm was validated through a series of laboratory measurements. The calibration cube was initially located on a flat surface of a CMM measurement machine (LK Metrology - LK Ultra 10.7.6) and the theoretical contact points of the dial indicators (Mitutoyo 2330S-10) were marked on the cube and measured for an aligned position of the cube. Then the cube was displaced by know amount of translations and rotations and the new position of the contact point were measured again and compared with the results of the algorithm. This method was used as a proof-of-concept for assessing the accuracy of the algorithm for the proposed forward model.

Subsequently, a second algorithm was developed to reverse the forward model and calculate the displacement values based on the indicators' contact points with the cube. However, for the inverse model, numerical methods had to be used, due to the number of unknown variables, to estimate the displacement values based on the comparison between contact points at $\phi$ and $-2 \phi$ degrees. The inverse model was validated in two ways. Firstly using the forwards model within an acceptable margin of error, and secondly, by testing at a shop floor rig and offsetting a robot coordinate based on the values from inverse model.

\section{Determining the misalignments at $6 \mathrm{DoF}$}

Forward and inverse solutions[15], [27] were considered to determine the misalignment values by using the notion of cause and effect. The forward problems predict the unknown effects of known causes by the use of derived effect prediction models. For instance, the misalignment between a robot and the cube will cause changes in dial indicators. The inverse problems however, aim to find the unknown cause of a measured effect, such as finding the misalignment values $x, y, z, A, B, C$ based on the readings obtained from the dial indicators. In the proposed approach, the observed displacements of the digital indicators infer the physical transformation and rotational parameters that caused this displacement effect. Inverse problems are generally more difficult to solve than their associated forward problem and in some cases, impossible due to the number of unknown variables. In these cases, approximation methods could be deployed.

The comparison between a theoretical perfect aligned system and the actual displaced system gives the transform ${ }_{D}^{A} T$ between the actual and perfect coordinates (in WORLD coordinate) and the actual displaced coordinate, from which one can determine ${ }_{D}^{A} T$ through the relationship given by Equation 
1. Using this equation, one can determine the 6 coordinate values of $x, y, z, A, B, C$ needed to define the offset between the ROBROOT and the WORLD coordinate systems.

$$
{ }_{R}^{A} T{ }_{R}^{D} T^{-1}={ }_{D}^{A} T
$$

Equation 1

Six measurements will be needed to determine the coordination of the cube at 6 DOF.Therefore the measurement disk will undergo two rotations in order to obtain two sets of three measurements. This mathematical problem falls into a "determined" problem by the number of unknowns/degrees of freedom in the problem and the number of constraints/equations. At least six constraints are necessary to remove each of 6 degrees of freedom. The six measurements lead to the six equations necessary to constrain the problem fully.

Consider the calibration cube shown by Figure 4, with edge length of $2 L$, has its centre at point ${ }^{\mathrm{D}}(0,0,0)$ with respect to its own reference frame, and has faces perpendicular to the coordinate axes. It is in the perfectly aligned position Palign such that the displaced WORLD frame $D$ coincides with the initial WORLD frame $W$. Three high precision dial indicators, attached to the measurement disk, are mounted on the tool centre point of the robot arm, which has the ability to rotate its tool centre point to both directions around the $v$ vector.

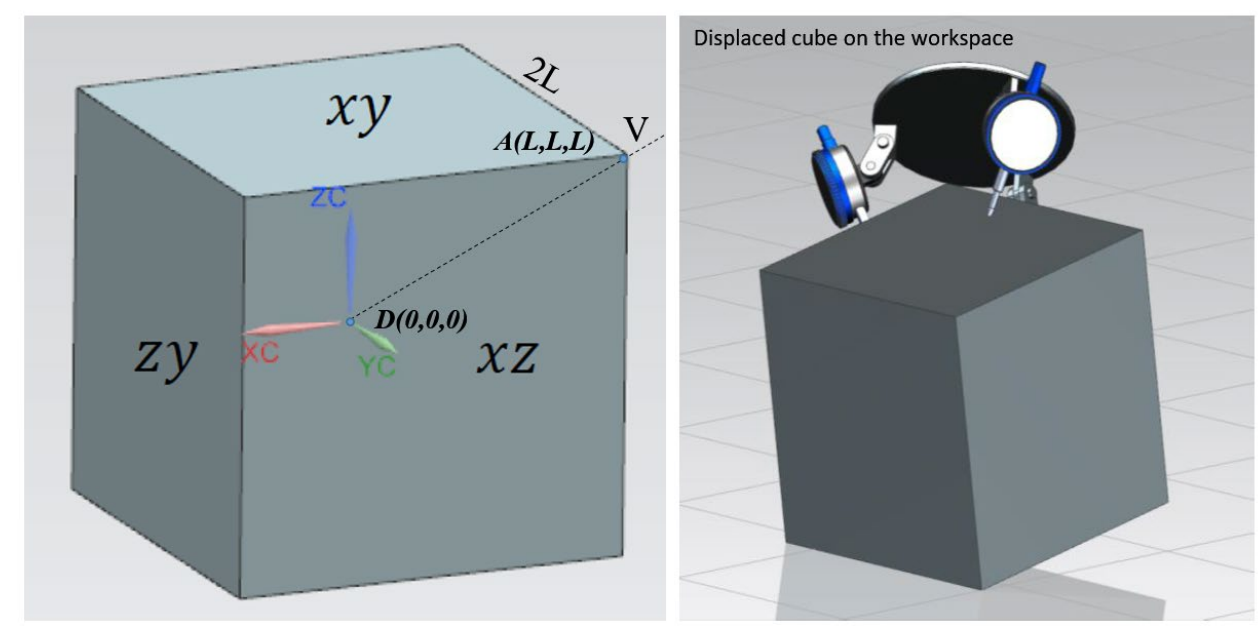

Figure 4: Measuring the misalignment on displaced cube

The robot arm is positioned in a manner such that the robot tool centre point is at the corner of the cube and its axis of rotation coinciding with the axis $V$ that passes through the point ${ }^{\mathrm{A}}(\mathrm{L}, \mathrm{L}, \mathrm{L})$ and the origin ${ }^{\mathrm{D}}(0,0,0)$. At such a position, the indicators touch each face meeting at the corner $A$. The measurement disk is designed so that each indicator is aligned to measure displacement in a direction such that their lines of action intersect at a point, somewhere along the axis $\boldsymbol{V}$, and intersect the diagonals running into the corner, along the relevant faces.

The tool centre point is then rotated through an angle $\phi$ around vector $V$ to give the initial measuring position $P_{1}$. Three measurements are obtained before the tool centre point is rotated again by $-2 \phi$ to give a second measuring position $P_{2}$.

This rotation permits 6 indicator measurements of $M_{x y 1}^{A}, M_{x y 2}^{A}, M_{z y 1}^{A}, M_{z y 2}^{A}, M_{x z 1}^{A}, M_{x z 2}^{A}$ to be taken with three measuring probs. $M_{x y n}^{A}$ indicates the measurements for a perfectly aligned coordinate, which robot was programmed to move to, and $M_{x y n}^{D}$ denotes the measurement taken from the dial at a the assumed displaced position.

From the definition given earlier, the forward model calculates the effect of a known displacement of the cube. The displacement of the three indicators at both left and right rotations can be calculated from a knowledge of the physical parameters of the system, i.e. the three rotations and the three 
translations. The six indicator displacements can then be defined by $\Delta M_{x y 1}, \Delta M_{x y 2}, \Delta M_{z y 1}, \Delta M_{z y 2}, \Delta M_{x z 1}, \Delta M_{x z 2}$, determined by Equation 2.

$$
\begin{aligned}
& \Delta M_{x y 1}=\left|{ }^{A} P_{x y 1}^{\text {align }}-{ }^{A} P_{x y 1}^{\text {displaced }}\right| \\
& \Delta M_{x y 2}=\left|{ }^{A} P_{x y 2}^{\text {align }}-{ }^{A} P_{x y 2}^{\text {displaced }}\right|
\end{aligned}
$$

$\Delta M_{x y 1}$ and $\Delta M_{x y 2}$ indicate the difference between the observed values of one dial indicator on one face, where ${ }^{A} P_{x y n}^{\text {align }}$ is the coordinate position of the intersection of dial indicator 1 on face $x y$, when the cube is in perfectly aligned position.

To solve the above forward problem, the points of intersection between each indicator and its respective surface in the aligned and disturbed positions must be evaluated by determining the LinePlane intersection equation. A general point on a line is given by Equation 3.

$$
l=l_{a}+\left(l_{b}-l_{a}\right) t, \quad t \in R
$$

Equation 3

Where $l_{a}=\left(x_{a}, y_{a}, z_{a}\right)$ and $l_{b}=\left(x_{b}, y_{b}, z_{b}\right)$ are two distinct points along a line, as shown by Figure 5 .

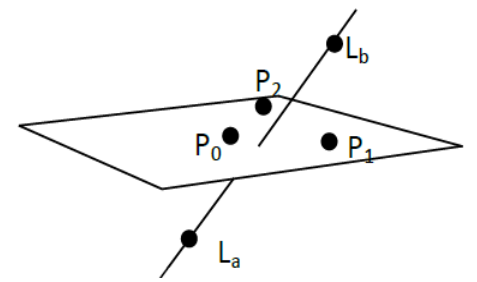

Figure 5: Intersection of a line and a plane

Similarly a general point on a plane is given by Equation 4,

$$
p=p_{0}+\left(p_{1}-p_{0}\right) u+\left(p_{2}-p_{0}\right) v, \quad u, v \in R
$$

Where $p_{k}=\left(x_{k}, y_{k}, z_{k}\right), \quad \mathrm{k}=0,1,2$ are three points on the plane, which are not collinear.

The point at which the line intersects the plane is therefore described by setting the point on the line equal to the point on the plane, giving the parametric Equation 5 :

$$
l_{a}-p_{0}=\left(l_{a}-l_{b}\right) t+\left(p_{1}-p_{0}\right) u+\left(p_{2}-p_{0}\right) v
$$

Equation 5

Which can be expressed in matrix form as shown by Equation 6 to solve $t, u$ and $v$.

$$
\left[\begin{array}{c}
t \\
u \\
v
\end{array}\right]=\left[\begin{array}{lll}
x_{a}-x_{b} & x_{1}-x_{0} & x_{2}-x_{0} \\
y_{a}-y_{b} & y_{1}-y_{0} & y_{2}-x_{0} \\
z_{a}-z_{b} & z_{1}-z_{0} & z_{2}-x_{0}
\end{array}\right]^{-1}\left[\begin{array}{l}
x_{a}-x_{0} \\
y_{a}-y_{0} \\
z_{a}-z_{0}
\end{array}\right]
$$

If the solution satisfies the condition $t \in[0,1]$ then the intersection point is on the line between $l_{a}$ and $l_{b}$. If the solution satisfies $u, v \in[0,1],(u+v) \leq 1$ then the intersection point is in the plane inside the triangle spanned by the three points $p_{0}, p_{1}$ and $p_{2}$.

For simplicity, the above equation is changed to Equation 7. 


$$
\begin{aligned}
& {\left[\begin{array}{ccc}
x_{a}-x_{b} & x_{1}-x_{0} & x_{2}-x_{0} \\
y_{a}-y_{b} & y_{1}-y_{0} & y_{2}-x_{0} \\
z_{a}-z_{b} & z_{1}-z_{0} & z_{2}-x_{0}
\end{array}\right]^{-1}=\left[\begin{array}{lll}
A & B & C \\
D & E & F \\
G & H & I
\end{array}\right]^{-1}} \\
& {\left[\begin{array}{l}
t \\
u \\
v
\end{array}\right]=\frac{\left[\begin{array}{ccc}
E I-F H & C H-B I & B F-C E \\
F G-D I & A I-C G & C D-A F \\
D H-E G & B G-A H & A E-B D
\end{array}\right]\left[\begin{array}{l}
x_{a}-x_{0} \\
y_{a}-y_{0} \\
z_{a}-z_{0}
\end{array}\right]}{(A)(E I-F H)-(B)(D I-F G)+(C)(D H-E G)}}
\end{aligned}
$$

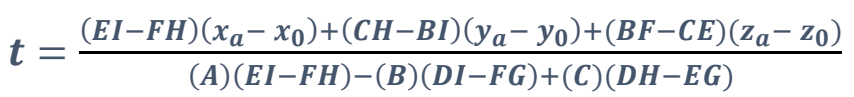

On substitution of $t$ into Equation 3 , we get the point of intersection $i$ of the line containing points $l_{a}$, and $l_{b}$, and the plane contacting points $p_{0}, p_{1}$ and $p_{2}$.

It follows that two points are needed on each line of action of the six dial indicators. Due to the geometry of the measurement disk, all indicator lines of action intersect at a common coordinate that lies arbitrarily along the axis $V$, which has a direction vector of $\vec{V}=\vec{\imath}+\vec{\jmath}+\vec{K}$.

Furthermore, each line intersects the circumference of a circle of radius $r$, perpendicular to and centred around the axis $V$. The second point required for each line can thus be described by the equation for this circle, as illustrated by Figure 6 .

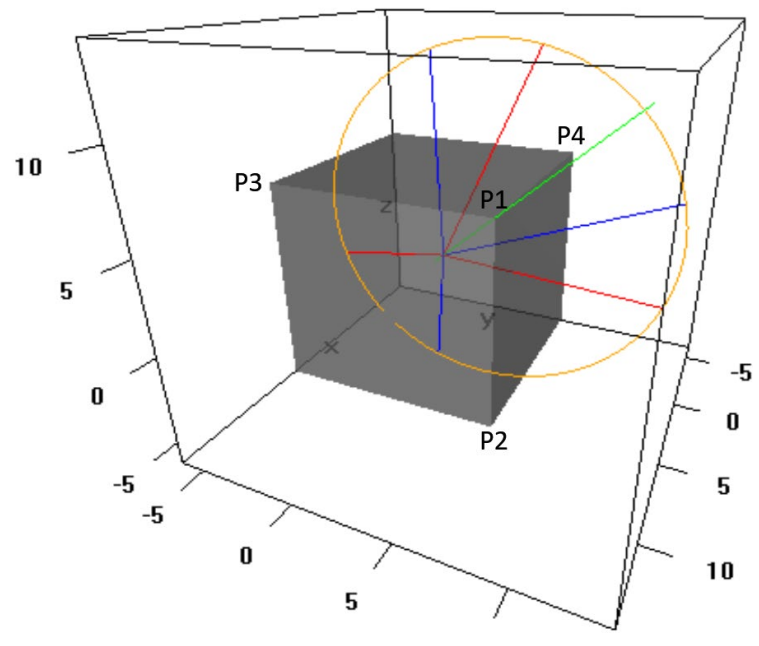

10

Figure 6 : The lines of intersections before and after rotation of the measurement disk

Assuming $\widehat{a}, \hat{b}$ and $\vec{v}$ are orthogonal vectors and point $\left(\begin{array}{l}c_{1} \\ c_{2} \\ c_{3}\end{array}\right)$ is any point along the axis $\vec{V}$, about which we wish to sketch the circle of radius $r$, then any point on the circle can be specified by the parametric Equation 8.

$$
\begin{aligned}
x(\theta)= & c_{1}+a_{1} r \cos \theta+b_{1} r \sin \theta \\
y(\theta)= & c_{2}+a_{2} r \cos \theta+b_{2} r \sin \theta \\
z(\theta)= & c_{3}+a_{3} r \cos \theta+b_{3} r \sin \theta \\
& 0 \leq \boldsymbol{\theta} \leq 2 \pi
\end{aligned}
$$


We further require to determine the planes that the dial indicators are intersecting with. Three noncollinear points are required for each $x y, z y$, and $x z$ planes, considering points $P_{1}, P_{2}, P_{3}, P_{4}$ (see Figure 6 ) and $l$ as the line between the circle and the centre of the cube.

When the cube is in a perfect aligned position (i.e. reference $A$ ), the points are determined by Equation 9.

$$
{ }^{A} P_{1}^{\text {align }}=\left[\begin{array}{c}
L_{x} \\
L_{y} \\
L_{z}
\end{array}\right],{ }^{A} P_{2}^{\text {align }}=\left[\begin{array}{c}
L_{x} \\
-L_{y} \\
L_{z}
\end{array}\right],{ }^{A} P_{3}^{\text {align }}=\left[\begin{array}{c}
-L_{x} \\
L_{y} \\
L_{z}
\end{array}\right],{ }^{A} P_{4}^{\text {align }}=\left[\begin{array}{c}
L_{x} \\
L_{y} \\
-L_{z}
\end{array}\right]
$$

When the cube is in the displaced position $P^{\text {displaced }}$, the coordinates of the four points with respect to the aligned position are found by pre-multiplying each point by the homogeneous transformation matrix ${ }_{D}^{A} T$. This transforms all points with respect to frame Aligned $(A)$ to the same points with respect to frame Displaced $(D)$, as defined by Equation 10.

$$
\begin{gathered}
{ }^{A} P_{n}^{\text {Displaced }}={ }_{D}^{A} T \quad{ }^{D} P_{n}^{\text {Displaced }} \\
\text { Where }{ }_{D}^{A} \boldsymbol{T}=\left[\begin{array}{cccc}
{ }^{A} R_{D} & { }^{A} P_{D} \\
0 & 0 & 0 & 1
\end{array}\right]
\end{gathered}
$$

Here ${ }^{W} R_{D}$ represents the general rotation matrix, which results from rotations about the $x, y$, and $z$ axes of the initial WORLD frame, to re-orientate the perfectly aligned cube to the displaced cube orientation.

The general rotation matrix represents a rotation about $z$, then $y$, and then $x$, determined by Equation 11.

$$
\begin{gathered}
{ }^{A} \boldsymbol{R}_{D}=\boldsymbol{R}_{x}(\gamma), \boldsymbol{R}_{\gamma}(\boldsymbol{\beta}), \boldsymbol{R}_{Z}(\boldsymbol{\alpha}), \text { when } \\
\boldsymbol{R}_{x}(\gamma)=\left[\begin{array}{ccc}
1 & 0 & 0 \\
0 & C_{\gamma} & -S_{\gamma} \\
0 & S_{\gamma} & C_{\gamma}
\end{array}\right], \boldsymbol{R}_{y}(\boldsymbol{\beta})=\left[\begin{array}{ccc}
C_{\beta} & 0 & S_{\beta} \\
0 & \mathbf{1} & \mathbf{0} \\
-S_{\beta} & \mathbf{0} & C_{\beta}
\end{array}\right], \boldsymbol{R}_{Z}(\boldsymbol{\alpha})=\left[\begin{array}{ccc}
C_{\alpha} & -S_{\alpha} & 0 \\
S_{\alpha} & C_{\alpha} & 0 \\
\mathbf{0} & 0 & 1
\end{array}\right] \\
{ }^{A} \boldsymbol{P}_{D}^{\text {Displaced }}=\left[\begin{array}{l}
x \\
y \\
Z
\end{array}\right]
\end{gathered}
$$

${ }^{A} P_{D}$ represents the position vector to the origin of the displaced frame with respect to the initial WORLD frame $A$. It is essentially the $x, y, z$ translation needed to move the centre of the aligned cube to the centre of the displaced cube.

Points of intersection of each dial indicator with each of the 3 planes can now be evaluated by specifying six points before and six points after rotating the measurement disk.

The points are calculated for each plane, which are 12 points in total. For instance, the points of intersection on plane $x y$ are ${ }^{A} i_{x y 1}^{\text {Align }},{ }^{A} i_{x y 1}^{\text {Displaced }},{ }^{A} i_{x y 2}$ Align,${ }^{A} i_{x y 2}^{\text {Displaced }}$.

Using the intersection points, the proposed forward model was developed in RStudio ${ }^{+}$[28] to predict the misplacement of the contact points (i.e. the readings from the dial indicators).

\footnotetext{
${ }^{+}$RStudio is a free and open-source integrated development environment (IDE) for R, a programming language for statistical computing and graphics[28].
} 
In this program, the user inputs five geometrical constants including: half cube length $l$, circle radius $r$, centre of circle, offset angle $p h i$, and point of indicator line-of-action intersection. In addition, 6 displacement variables $(x, y, z, \alpha, \beta, \gamma)$ are introduced, which constrain the cube in six degrees of freedom. The programme therefore calculates the displacement of the dials' contact points with the cube surface.

The plotting feature of the RStudio was used to plotting a 3D diagram of the model shown in Figure 6, using the function plotVerify $<-$ function (l,radius,centre,phi,Probe, $x, y, z,, y)$ ).

\subsection{Validating the forward model}

In addition to the graphical presentation, a laboratory test was carried out to evaluate the algorithms used for the forward model. A set of arbitrary geometrical values were given to the algorithm according to the geometry of the test rig, as shown by Table 1 . The test rig was simply a titling table mounted on a CMM machine, holding the calibration cube at a certain known displaced position.

Table 1: Geometrical constants used for the evaluation of the forward model

\begin{tabular}{|l|l|}
\hline Half cube length, I & 6 \\
\hline Circle radius, $r$ & 7 \\
\hline Coordinates of centre of circle & 8 \\
\hline Probe offset angle & 20 \\
\hline Coordinate of probe line of action intersection & 1 \\
\hline
\end{tabular}

The plotted diagrams are illustrated by Figure 7. A perfectly alighted position was specified by parametric values of $(0,0,0,0,0,0)$, and the displaced position was specified by $(2,1,3,10,5,15)$ values.

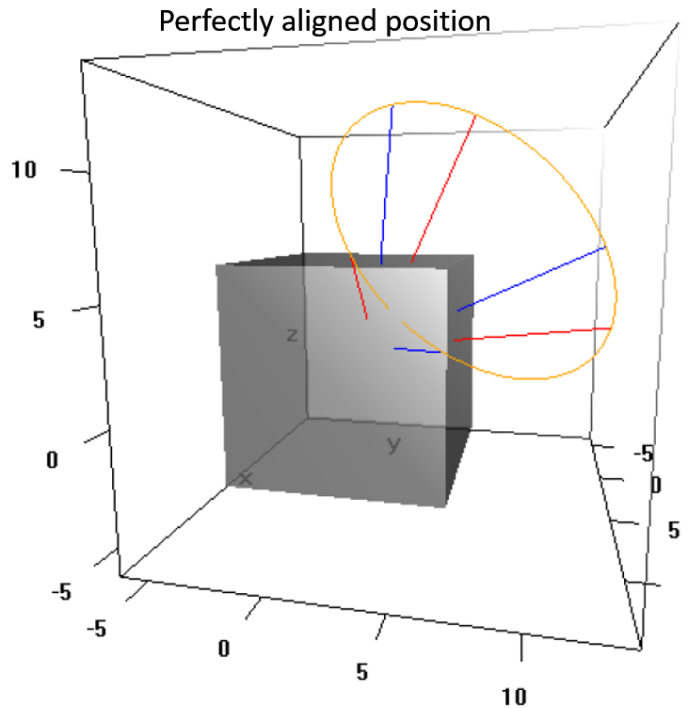

Gamma $=0$ degrees $\quad \times$ Translation $=0$ units Beta $=0$ degrees $\quad$ Y Translation $=0$ units Alpha $=0$ degrees $z$ Translation $=0$ units

Displacment $X Y 1=0$ units $\quad$ Displacment $X Y 2=0$ units Displacment $X Z 1=0$ units

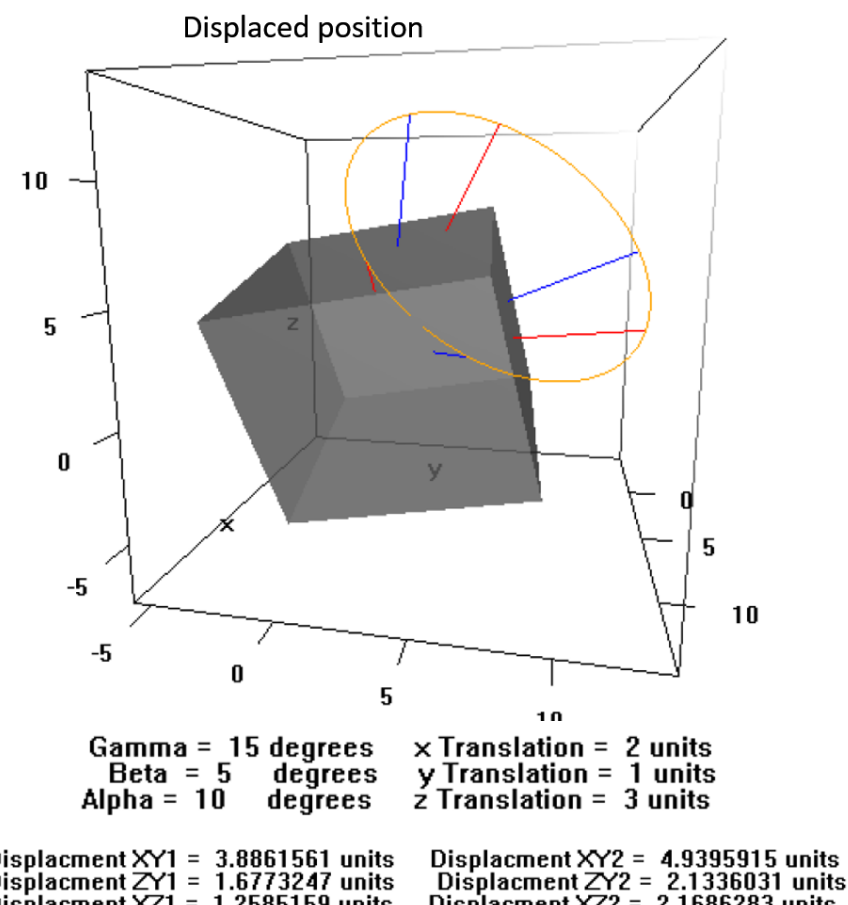

Displacment $Z Y 1=1.6773247$ units Displacment $Z Y 2=2.1336031$ unit

Figure 7: A forward model to calculate the coordinates of the dials' contact points

As shown in the figure, the program returns positive displacements for each probe at displaced position, indicating that each probe contracts in length by the specified amount. Furthermore, by plotting a line that starts from the circle intersection point and terminates at the calculated intersection of the line and plane, it can be seen from visual inspection that the calculated point of 
intersection does in fact lie on the face of the cube. This observation was examined by the measurement of the cube in laboratory, which validated the precision of the forward model.

In this programme a negative number returned value for any displacement indicates that the dial indicator in question expands in length.

\subsection{The inverse problem}

A model for solving the above forward problem was derived based on the six evaluations of an explicit equation, in which dependant variable $l_{n}$ was expressed explicitly as a function, albeit of a nonlinear nature, of the dependant variables $x, y, z, \alpha, \beta, \gamma$. Therefore:

$$
\begin{gathered}
x, y, z, \alpha, \beta, \gamma \rightarrow\left[\begin{array}{lll}
\mathrm{x}_{\mathrm{a}}-\mathrm{x}_{\mathrm{b}} & \mathrm{x}_{1}-\mathrm{x}_{0} & \mathrm{x}_{2}-\mathrm{x}_{0} \\
\mathrm{y}_{\mathrm{a}}-\mathrm{y}_{\mathrm{b}} & \mathrm{y}_{1}-\mathrm{y}_{0} & \mathrm{y}_{2}-\mathrm{x}_{0} \\
\mathrm{z}_{\mathrm{a}}-\mathrm{z}_{\mathrm{b}} & \mathrm{z}_{1}-\mathrm{z}_{0} & \mathrm{z}_{2}-\mathrm{x}_{0}
\end{array}\right]^{-1}\left[\begin{array}{l}
\mathrm{x}_{\mathrm{a}}-\mathrm{x}_{0} \\
\mathrm{y}_{\mathrm{a}}-\mathrm{y}_{0} \\
\mathrm{z}_{\mathrm{a}}-\mathrm{z}_{0}
\end{array}\right]=\left[\begin{array}{l}
\mathrm{t} \\
\mathrm{u} \\
\mathrm{v}
\end{array}\right] \rightarrow t\left(l_{b}-l_{a}\right)+l_{a}=l_{n} \\
\frac{l_{n}-l_{a}}{l_{b}-l_{a}}=\boldsymbol{t} \rightarrow l_{n}=\boldsymbol{f}(\boldsymbol{x}, \boldsymbol{y}, \mathrm{z}, \boldsymbol{\alpha}, \boldsymbol{\beta}, \gamma)
\end{gathered}
$$

As a starting point to the above inverse problem, the reverse of the forward problem was considered. With the knowledge of six points of intersection, six values of $t_{n}$ (n from 1 to 6 ) are evaluated from Equation 12. The substitution of these values into Equation 6 results in a nonlinear system of 6 implicit equations as shown by Equation 13 .

$$
\boldsymbol{t}_{n}=\boldsymbol{f}(\boldsymbol{x}, \boldsymbol{y}, \boldsymbol{z}, \boldsymbol{\alpha}, \boldsymbol{\beta}, \gamma) \quad \boldsymbol{n} \text { from } 1 \text { to } 6
$$

Equation 13

The nonlinearity of this equation arises from the fact that three of the unknowns appear as arguments of trigonometric functions. Unlike a linear system, which is analytically solvable and has a unique solution, nonlinear systems can have several solutions, and only very simple cases can be solved analytically. Therefore, iterative numerical techniques[15], [29], such as the Newton-Raphson algorithm [30], [31] were used to solve this problem. These techniques use numerical approximations to solve the problems. Unlike a direct method that computes an approximate solution to a problem in a finite number of steps, in the iterative method used in this experiment the algorithm terminates after a finite number of steps. The numerical approximation was initiated with the values of the aligned position of the measurement cube. Our proposed algorithm makes successive approximations that converge on the exact solution at infinity. However, it is significantly faster for our required accuracy limit, and therefore only a finite number of iterations are required in order to reach a solution of sufficient accuracy.

Using RStudio once again, an algorithm for the proposed Inverse Model was developed to implement the method used to return the model parameters $x, y, z, \alpha, \beta, \gamma$ from the observed displacement of the dial indicators, i.e. $\Delta M_{x y 1}, \Delta M_{x y 2}, \Delta M_{x z 1}, \Delta M_{x z 2}, \Delta M_{z y 1}, \Delta M_{z y 2}$. The proposed algorithm can be broken down into three stages. At the first stage, the conversion of the displacement $\Delta M_{n}$ to $l_{n}$ and the point of intersection of probe $\mathrm{n}$ and the cube in the displaced position are carried out. This conversion process is done using the vector addition: $l_{n}=l_{p}+\Delta M_{n} \cdot \hat{u} \hat{p}_{p \rightarrow n}$ where $l_{p}$ is the point of intersection of probe $n$ and the cube in the perfectly aligned position, and $\hat{u}_{p \rightarrow n}$ is a unit vector in the same direction as the line of action of probe $\mathrm{n}$, in the sense of $l_{p}$ to $l_{n}$. At the following stage, $t_{n}$ is evaluated from Equation 13 , where $l_{a}$ and $l_{b}$ are evaluated as in the forward problem. This is a point-wise algebraic substitution to evaluate $t_{n}$, forming the nonlinear system on substitution into Equation 12.

At the final stage, the Newton-Raphson method is used to solve $0=f\left(t_{n}, x, y, z, \alpha, \beta, \gamma\right)$. 
In this method, to find the roots of nonlinear functions (where $f(x)=0$ ) the convergence is quadratic, i.e. the number of accurate digits is roughly doubled after each iteration. Given an initial approximation of $x_{0}$, successive iterations are computed using Equation 14.

$$
x_{n+1}=x_{n}-\frac{f\left(x_{n}\right)}{f^{\prime}\left(x_{n}\right)}
$$

Equation 14

For any $f(x)$ for which $f(\propto)=0$, the Newton's method will converge under three conditions:

a) the initial $x_{0}$ should be sufficiently close to the real root $\propto$,

b) $f^{\prime}(\mathrm{x}) \neq 0 ; \forall x \in I$, where $\mathrm{I}$ is the interval $[\propto-r, \propto+r]$ for some $r \geq\left|\left(\alpha-x_{0}\right)\right|$,

c) $f^{\prime \prime}\left(x_{n}\right)$ is finite, $\forall x \in I$.

Therefore, by redefining Equation 13 as $F_{n}=f\left(t_{n}, x, y, z, \alpha, \beta, \gamma\right)$, it was found that the values of $t_{n}$, $F_{n}$ meets second and third conditions, when $x_{0}, y_{0}, z_{0}, \alpha_{0}, \beta_{0}, \gamma_{0}=0$ are consider at starting point.

With the six functions defined from Equation 12, the function multiroot of the rootSolve feature in the RStudio package was used to estimate the root of the equations to a user specified accuracy, which returns the six model parameters (i.e. the displacement values at 6 DoF), given the six indicator displacement values.

\section{Validating the inverse model}

To test the accuracy of the inverse model, two methods were used. The forward model was initially used to evaluate the inverse model for known displacement values. The displacement values of the digital indicators were predicted using the forward model. These predictions are then used as input arguments for the inverse model, which should in theory return the same model parameters used as the input arguments in the forward model. Table 2 shows the result of two series of tests between the forward and inverse models.

Table 2: Test results for 2 degrees rotation of the disk

\begin{tabular}{|c|c|c|c|c|c|c|c|}
\hline \multicolumn{4}{|c|}{ Forward Model } & \multicolumn{4}{|c|}{ Inverse Model } \\
\hline \multicolumn{2}{|c|}{ Input Values } & \multicolumn{2}{|c|}{ Calculated Values } & \multicolumn{2}{|c|}{ Input Values } & \multicolumn{2}{|c|}{ Calculated Values } \\
\hline \multicolumn{8}{|c|}{ Test (1) } \\
\hline$x$ & -1.783 & $\Delta x y_{1}$ & 0.356 & $\Delta x y_{1}$ & 0.352 & $x$ & -1.784 \\
\hline$y$ & 1.252 & $\Delta x y_{2}$ & 0.805 & $\Delta x y_{2}$ & 0.803 & $y$ & 1.249 \\
\hline$z$ & 2.034 & $\Delta x z_{1}$ & 2.021 & $\Delta x z_{1}$ & 2.020 & $z$ & 2.034 \\
\hline$\alpha$ & 5.676 & $\Delta x z_{2}$ & 2.116 & $\Delta x z_{2}$ & 2.125 & $\alpha$ & 5.676 \\
\hline$\beta$ & 21.476 & $\Delta z y_{1}$ & -1.727 & $\Delta z y_{1}$ & -1.731 & $\boldsymbol{\beta}$ & 21.473 \\
\hline$\gamma$ & -4.914 & $\Delta z y_{2}$ & -1.109 & $\Delta z y_{2}$ & -1.110 & $\gamma$ & -4.920 \\
\hline \multicolumn{8}{|c|}{ Test (2) } \\
\hline$x$ & -0.056 & $\Delta x y_{1}$ & 1.609 & $\Delta x y_{1}$ & 1.609 & $x$ & -0.056 \\
\hline$y$ & 0.345 & $\Delta x y_{2}$ & 1.869 & $\Delta x y_{2}$ & 1.868 & $y$ & 0.346 \\
\hline$z$ & 1.255 & $\Delta x z_{1}$ & 0.293 & $\Delta x z_{1}$ & 0.293 & $z$ & 1.257 \\
\hline$\alpha$ & 3.039 & $\Delta x z_{2}$ & 0.554 & $\Delta x z_{2}$ & 0.554 & $\alpha$ & 3.039 \\
\hline $\boldsymbol{\beta}$ & 1.09 & $\Delta z y_{1}$ & -0.227 & $\Delta z y_{1}$ & -0.226 & $\beta$ & 1.092 \\
\hline$\gamma$ & 6.003 & $\Delta z y_{2}$ & -0.126 & $\Delta z y_{2}$ & -0.125 & $\gamma$ & 6.010 \\
\hline
\end{tabular}

$\Delta x y=$ contraction or extension of the dial indicators Calculated values are rounded up to 4 decimal places 
As illustrated by Table 2 at the highlighted row in test 1 for example, for a defined configuration on the rig, the $z$ value of a known misalignment was measured at $2.034 \mathrm{~mm}$, which according to the forward model, would generate a $2.0203506 \mathrm{~mm}$ extension on the dial indicator at the $x z$ plane. This value was also tested by the inverse model and the $z$ value was calculated as $2.034424 \mathrm{~mm}$, which it is within the acceptable margin of errors. This method was used to assess the reliability of the numerical estimations used in the inverse model.

These two examples however serve only as a proof of method, rather than a validation of practicality. Up to this stage, the input design parameters of the models have been arbitrarily chosen based on suitability for the laboratory test rig. However, the design limitations imposed by the range and precision of the digital indicators should also be considered in this method. For instance, there are limitations with the size of work piece that can be sufficiently aligned by the proposed method due to the restrictions in measuring range of the digital indicators. In the following sections, the limitations and the practical application of the approach are discussed.

\section{Application constraints}

In this exercise, a robot arm (KUKA KR 16-2) was used as the manipulator to which the workpiece is intended to be aligned to. This robot has a repeatability of $50 \mu \mathrm{m}$ that indicates the precision of the robot capability to move to a defined position repeatedly. Hence, it is necessary to require every point on the workpiece to be within of its position in the CAD model used for the programming. Therefore, it can be said that the Inverse model must specify translations accurate to at least this value. More thought however must be made with regard to the angular accuracy that must also be specified.

The typical probe indicators applicable for this application domain have the ability to measure displacements to a precision of $1 \mu \mathrm{m}$ theoretically (in practice, this value may change based on the environment and the quality of equipment). Since this is $\frac{1}{50}$ th of the manipulator's repeatability range, it can be shown that the alignment cube can be up to $\frac{1}{50} t h$ of the size of the workpeice which is to be aligned, as shown in Figure 8.

However, there is in fact a limit to the size of the alignment cube that can be sufficiently aligned. Assuming a simplified case of rotational misalignment limited only to the $z$ axis, and translational misalignment limited only to the $\theta$ axis, as a misalignment scenario. It has previously been mentioned that the maximum expected translational misalignment is $3 \mathrm{~mm}$ and the maximum expected rotational misalignment is $5^{\circ}$. If an indicator is positioned a distance $x$ from the centre of rotation with its line of action perpendicular to the face in the aligned position it can be said that the indicator must have a minimum measuring range of $R \mathrm{~mm}$ as defined by Equation 15 .
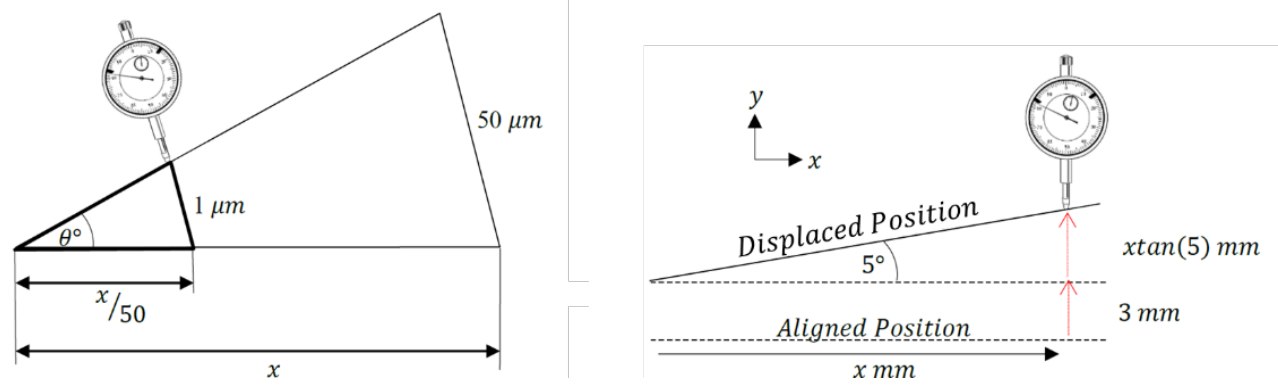

Figure 8: Specifying the size of the measurement equipment

$$
R \geq 2\left(3 m m+x \tan \left(5^{\circ}\right)\right) \rightarrow x \leq \frac{\left(\frac{R}{2}-3\right)}{\tan (5)}
$$


The proposed assumption for a 2DoF misalignment scenario provides an indication on how complex the calculation would be for a 6 DoF, if it was to be solved analytically. To simplify the problem, the data obtained from the forward model was analysed under various extreme misalignment scenarios and it was found that the indicators must be placed no more than $108 \mathrm{~mm}$ from the centre of the cube of $120 \mathrm{~mm}$ of dimension and a minimum of $24 \mathrm{~mm}$ extension to the dial indicator is required.

In addition, the value of rotation is constrained by the application issues such as the robot, indicator dials, and the minimum readable values from the dials. Through experiments value of $\pm 2^{\circ}$ was found appropriate for the rotation.

\section{Application experiments}

To evaluate the accuracy of the proposed approach a set of experiments on the robot were carried out to identify the precision of the inverse model proposed. A calibration cube of $120 \mathrm{~mm}$ in dimension was used on a workspace with the robot equipped with the measurement disk with the three indicators. The dimensional error of the cube was considered negligible following a laboratory measurement, and the initial state of the system was reset to zero values to be considered as the perfect aligned position. A very large number of experiments were carried out with combination of intended misalignments values at each 6 degrees of freedom. In these experiments, the misalignment values of $-3 \mathrm{~mm}, 0$, and $3 \mathrm{~mm}$ were imposed to the linear axes (i.e. $x, y, z$ ) and rotational values of -5 , 0,5 degrees were applied to the $\alpha, \beta$, and $\gamma$ axes. In total 729 tests were carried out to provide a reliable average of model accuracy. Three combinations of the maximum translational and rotational errors (i.e. $3 \mathrm{~mm}$ and $5^{\circ}$ ) are illustrated by Table 3 (e.g. in the test no 1 , the misalignment values of $x=-3, y=3, z=-3, \alpha=5, \beta=-5, \gamma=-5$ were applied to the measurement cube). The robot was programmed to move to the assumed perfect aligned position of the cube within the workspace and after contacting the dials with the cube, robot rotated 2 degrees to the both directions. The third column of the Table 3 indicates the values calculated by the inverse model after inputting the 6 dial indicator readings observed. The results of three tests are illustrated by the table that indicate an accuracy of $0.003 \mathrm{~mm}$ for the translational misalignment and $0.002^{\circ}$ for the rotational misalignment. These values are confidently within the $0.05 \mathrm{~mm}$ repeatability range of the robot.

Based on the test configuration and the results, it is calculated that a workpiece of up to $1.7 \mathrm{~m}$ length can be aligned within the acceptable range of $0.01 \mathrm{~mm}$ for a typical automation system.

Table 3: Evaluating the precision of the proposed system on a robot

\begin{tabular}{|l|r|r|r|}
\hline & Actual Misalignment & Predicted Misalignment & Model Accuracy $( \pm)$ \\
\hline$x$ & $-3.0000 \mathrm{~mm}$ & $-3.00104 \mathrm{~mm}$ & $0.002 \mathrm{~mm}$ \\
\hline$y$ & $3.0000 \mathrm{~mm}$ & $3.00208 \mathrm{~mm}$ & $0.002 \mathrm{~mm}$ \\
\hline$Z$ & $-3.0000 \mathrm{~mm}$ & $-3.00046 \mathrm{~mm}$ & $0.0005 \mathrm{~mm}$ \\
\hline$\alpha$ & $5.0000^{\circ}$ & $4.99814^{\circ}$ & $0.002^{\circ}$ \\
\hline$\beta$ & $-5.0000^{\circ}$ & $-4.99992^{\circ}$ & $0.0001^{\circ}$ \\
\hline$\gamma$ & $-5.0000^{\circ}$ & $-4.99944^{\circ}$ & $0.001^{\circ}$ \\
\hline$x$ & $3.0000 \mathrm{~mm}$ & $3.00212 \mathrm{~mm}$ & $0.002 \mathrm{~mm}$ \\
\hline$y$ & $3.0000 \mathrm{~mm}$ & $2.99821 \mathrm{~mm}$ & $0.002 \mathrm{~mm}$ \\
\hline$z$ & $-3.0000 \mathrm{~mm}$ & $-3.00003 \mathrm{~mm}$ & $0.00003 \mathrm{~mm}$ \\
\hline$\alpha$ & $-5.0000^{\circ}$ & $-4.99899^{\circ}$ & $0.0001^{\circ}$ \\
\hline$\beta$ & $5.0000^{\circ}$ & $4.9979^{\circ}$ & $0.002^{\circ}$ \\
\hline$\gamma$ & $5.0000^{\circ}$ & $4.99827^{\circ}$ & $0.002^{\circ}$ \\
\hline$x$ & $3.0000 \mathrm{~mm}$ & $2.99698 \mathrm{~mm}$ & $0.003 \mathrm{~mm}$ \\
\hline$y$ & $-3.0000 \mathrm{~mm}$ & $-2.99965 \mathrm{~mm}$ & $0.0003 \mathrm{~mm}$ \\
\hline$Z$ & $3.0000 \mathrm{~mm}$ & $3.00224 \mathrm{~mm}$ & $0.002 \mathrm{~mm}$ \\
\hline$\alpha$ & $-5.0000^{\circ}$ & $-5.00193^{\circ}$ & $0.002^{\circ}$ \\
\hline$\beta$ & $5.0000^{\circ}$ & $5.00213^{\circ}$ & $0.002^{\circ}$ \\
\hline$\gamma$ & $5.0000^{\circ}$ & $4.99866^{\circ}$ & $0.001^{\circ}$ \\
\hline
\end{tabular}


However, the values $x, y, z, \alpha, \beta$, and $\gamma$ describe the position of the displaced WORLD frame with respect to the initial WORLD frame. In the application domain, this values should be mapped to the ROBROOT frame (see Figure 2) to complete the calibration process by offsetting the robot reference system. The homogeneous transform ${ }_{R}^{D} T$ can be determined from Equation 16.

$$
{ }_{R}^{A} T{ }_{D}^{A} T^{-1}={ }_{R}^{D} T
$$

Equation 16

Where ${ }_{R}^{A} T$ is determined from the initial offset values, and ${ }_{R}^{D} T$ is determined from $x, y, z, \alpha, \beta$, and $\gamma$. Then, it can be shown that

$$
{ }^{D} P_{R}=\left[\begin{array}{l}
x \\
y \\
z
\end{array}\right] \text { and } \quad{ }_{R}^{D} R=R(A, B, C)=\left[\begin{array}{lll}
r_{11} & r_{12} & r_{13} \\
r_{21} & r_{22} & r_{23} \\
r_{31} & r_{32} & r_{33}
\end{array}\right]
$$

Thus, the $X, Y$ and $Z$ translations can be extracted directly from ${ }_{R}^{D} T$. The offset rotations $A, B$, and $C$, however are determined from ${ }_{R}^{D} R$ by implementing a Euler angle extraction, when $R(A, B, C)$ first performs rotation around $X$, then $Y$, and finally $Z$.

A final RStudio script was developed to extract the offset angles from the rotation matrix, and has thus been combined with the Inverse model to form a complete cube alignment program.

A user interface for the algorithms were developed programme to facilitate of the implementation

\begin{tabular}{|c|c|c|c|c|c|}
\hline \multicolumn{3}{|l|}{ 啰 Cube Alignment } & & \multicolumn{2}{|c|}{\begin{tabular}{|l|l|l|}
$口$ & 回 & $x$ \\
\end{tabular}} \\
\hline \multicolumn{2}{|c|}{ Device Configuration } & & \multicolumn{3}{|c|}{$\begin{array}{l}\text { Initial ROBROOT offset } \\
\text { Position }\end{array}$} \\
\hline Cube Length & 120.00 & $\mathrm{~mm}$ & $\mathrm{x}$ & 0.00 & $\mathrm{~mm}$ \\
\hline Disk Radius & 105.00 & $\mathrm{~mm}$ & Y & 0.00 & $\mathrm{~mm}$ \\
\hline Indicator Angle & 20.00 & deg & Z & 0.00 & $\mathrm{~mm}$ \\
\hline Indicator Offset & 1.50 & deg & \multicolumn{2}{|c|}{ Orientation } & \multirow[b]{2}{*}{$\operatorname{deg}$} \\
\hline \multicolumn{2}{|l|}{ TOOL Origin } & & A & 0.00 & \\
\hline$x$ & 1620.00 & $\mathrm{~mm}$ & $B$ & 0.00 & deg \\
\hline$Y$ & 0.00 & $\mathrm{~mm}$ & C & 0.00 & deg \\
\hline \multirow[t]{2}{*}{ Z } & 1910.00 & $\mathrm{~mm}$ & & & \\
\hline & & & Reset & Mea: & asure Offset \\
\hline
\end{tabular}
process and illustrated Figure 9.

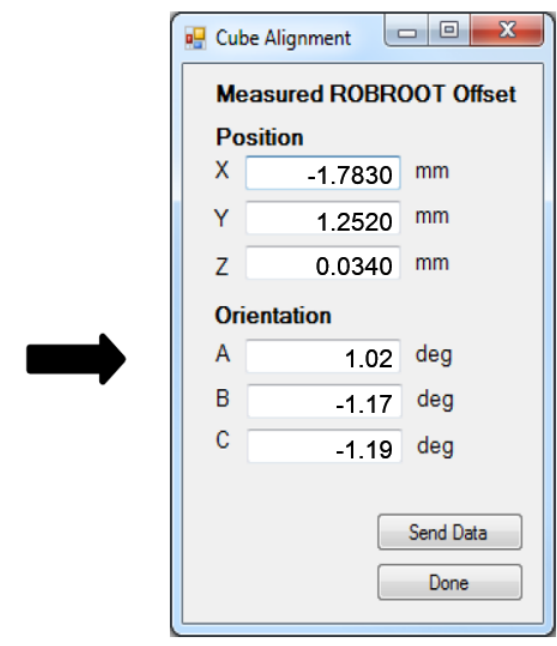

Figure 9: User interface to the inverse model

This interface was used for the experiments on the robot by manually inputting the values and receiving the robot offset needed to accommodate the misalignments values. However, if the digital indicators with automatic reading mechanism were used, the programme can be readily integrated into the robot input port to directly apply the robot offset to the robot positioning system as illustrated by Figure 10. In most windows based robot interfaces (e.g. the one used in this research), the computational process can be completed by the robot control unit, eliminating the need for additional computational system. 


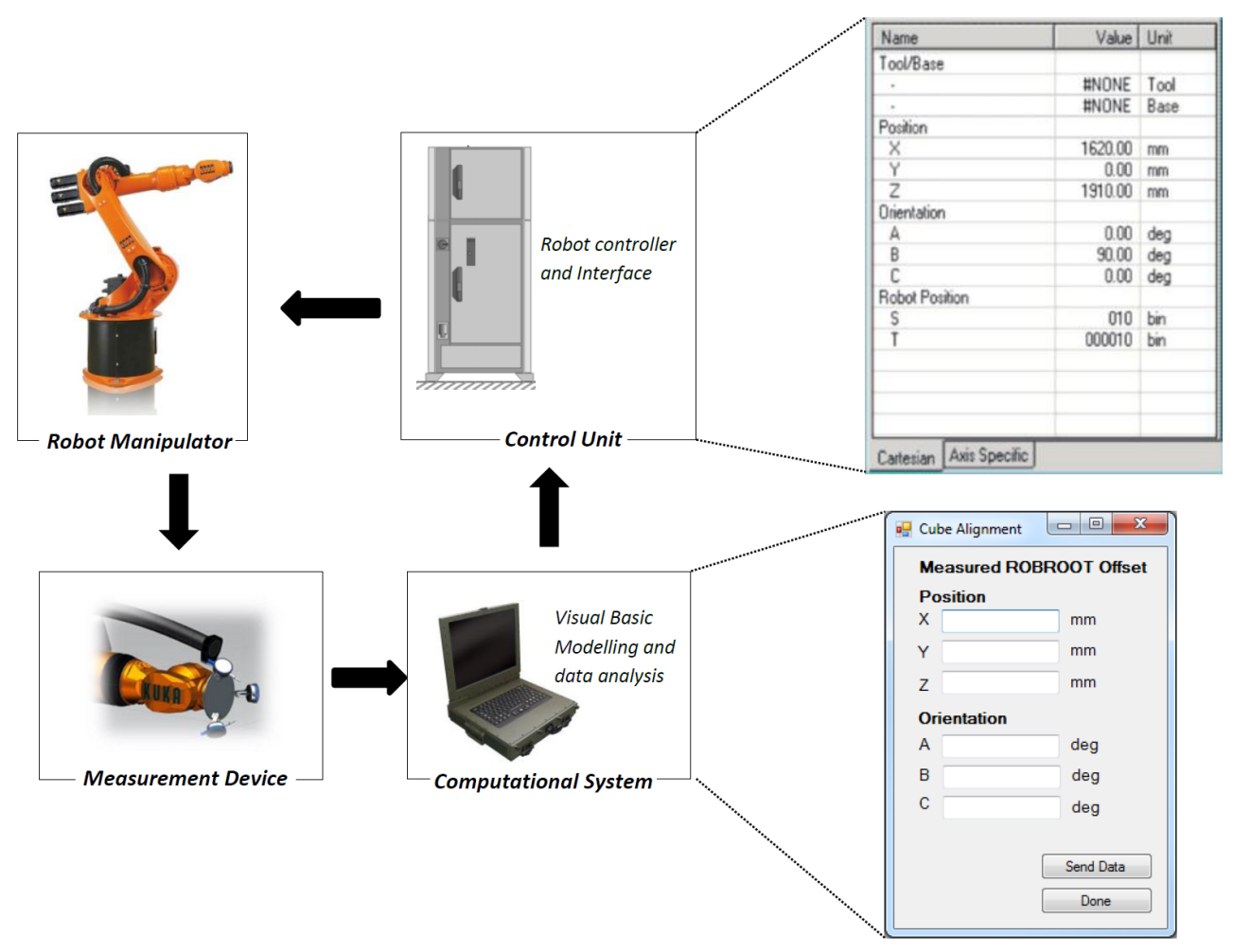

Figure 10: Integration of the misalignment offset to the robot controller

\section{Conclusion and further work}

In this article, the authors discussed a need for a low-cost high precision method to fully align robotic arms in an automation system. The existing methods were reviewed and highlighted that the low-cost manual methods are slow and skill-dependent, and high-end solutions provide unnecessarily high precision and are typically unaffordable for small manufacturers. A novel method of semi-automated alignment process was proposed in this article that allows adequate accuracy for the concerned domain of industry, with a very low investment cost. The novelty of the proposed approach is the use of simple design, supported by a series of complicated mathematical algorithms and the capability to be integrated into a robot control system.

The proposed method consists of three dial indicators mounted to a robotic arm and a measurement cube that locates on the workspace. A mathematical model was developed to calculate the changes on the dial indicators, if the cube locates in a known misaligned position. This model was tested using a laboratory measurement and was validated. A second algorithm was developed to reverse the first model and predict the misalignment parameters at 6 DoF, based on the reading of the dial indicators. This model was tested by comparing its results with the first validated model. Further practical experiments were carried out on a robot to estimate the accuracy of the second model.

It was found that the prediction model can estimate the misalignment with up to $0.003 \mathrm{~mm}$ and 0.002 degrees of error in translations and rotations consecutively. The accuracy range generated from this research is higher than what is required for this domain of industry.

Further research is planned to understand fully the influence of the potential deflection of the dial indicators and the impact of the initial assumption on the precision of the dials on the final alignment accuracy. This is to evaluate the robustness of the approach for a potential deployment in industrial domains. For this purpose, more experimental work is arranged to evaluate whether the system 
precision in various industrial set-ups is consistent and reliable as it is in laboratory environment, in particular with respect to the position of the cube relative to the workspace. Following this step, focus will be placed on the integration of the measured data to the robot control system automatically. This is to offset the robot positioning points based on the found misalignment values. Following a discussion with an industrial collaborator, the potential for a commercial solution is being considered as an add-on software to the existing robot control system.

\section{References}

[1] G. Wittenberg, "Developments in offline programming: an overview," Ind. Robot An Int. J., vol. 22, no. 3, pp. 21-23, Jun. 1995.

[2] H. Asada and J. Leonard, "Introduction to Robotics," 2010. [Online]. Available: http://ocw.mit.edu/courses/mechanical-engineering/2-12-introduction-to-robotics-fall2005/index.htm.

[3] R. Bernhardt, "Robots in CIM," in Integration of Robots into CIM, Dordrecht: Springer Netherlands, 1992, pp. 3-5.

[4] T. Takatsuji, M. Goto, A. Kirita, T. Kurosawa, and Y. Tanimura, "The relationship between the measurement error and the arrangement of laser trackers in laser trilateration," Meas. Sci. Technol., vol. 11, no. 5, pp. 477-483, May 2000.

[5] A. Korzun, E. Kukareko, and A. Pashkevich, "Estimation of robot parameters using optical sensors," 1993, vol. 2056, pp. 334-341.

[6] E. P. Kukareko, A. P. Pashkevich, D. E. Khmel, A. N. Korzun, and Y. L. Yurkewich, "Accuracy Increasing of Robot Real-Time Control," IFAC Proc. Vol., vol. 25, no. 20, pp. 177-180, Sep. 1992.

[7] W. Zhenhua, X. Hui, C. Guodong, S. Rongchuan, and L. Sun, "A distance error based industrial robot kinematic calibration method," Ind. Robot An Int. J., vol. 41, no. 5, pp. 439-446, Aug. 2014.

[8] Renishaw, "The Renishaw QC20-W wireless ballbar for machine tool performance diagnosis." Renishaw plc, 2013.

[9] G. T. Smith, Machine tool metrology: An industrial handbook. Springer, 2016.

[10] R. Bernhardt and S. L. Albright, Robot calibration. Chapman \& Hall, 1993.

[11] P. Bai, J. Mei, T. Huang, and D. G. Chetwynd, "Kinematic calibration of Delta robot using distance measurements," Proc. Inst. Mech. Eng. Part C J. Mech. Eng. Sci., vol. 230, no. 3, pp. 414-424, Feb. 2016.

[12] J. Maye, H. Sommer, G. Agamennoni, R. Siegwart, P. Furgale, R. Bernhardt, S. L. Albright, M. R. Driels, and U. S. Pathre, "Online self-calibration for robotic systems," IEEE Trans. Robot. Autom., vol. 35, no. 3, p. 311, Jun. 1991.

[13] M. R. Driels and U. S. Pathre, "Vision-based automatic theodolite for robot calibration," IEEE Trans. Robot. Autom., vol. 7, no. 3, pp. 351-360, Jun. 1991.

[14] "When it has to be right | Leica Geosystems." [Online]. Available: https://leicageosystems.com/en-gb. [Accessed: 05-Jun-2018].

[15] L. S. Ginani and J. M. S. T. Motta, "Theoretical and practical aspects of robot calibration with experimental verification," J. Brazilian Soc. Mech. Sci. Eng., vol. 33, no. 1, pp. 15-21, Mar. 2011.

[16] K. Lau, R. J. Hocken, and W. C. Haight, "Automatic laser tracking interferometer system for robot metrology," Precis. Eng., vol. 8, no. 1, pp. 3-8, Jan. 1986.

[17] A. M. Ivan, R. C. Parpală, L. Popa, and C. Coman, "EVALUATION OF ARTICULATED ARM ROBOT ACCURACY USING A LASER INTERFEROMETER," Proc. Manuf., vol. 12, no. 3, pp. 129-134, 2017. 
[18] J. C. Mullikin, L. J. van Vliet, H. Netten, F. R. Boddeke, G. van der Feltz, and I. T. Young, "Methods for CCD camera characterization\&lt;/title\&gt;," in SPIE 2173, Image Acquisition and Scientific Imaging Systems, 1994, vol. 2173, pp. 73-84.

[19] G. H. Prenninger JP, Vincze M, "Measuring Dynamic Robot Movements in 6 DOF and Real Time," in Robot Calibration, 1992.

[20] "Tech Sheet FARO Laser Tracker Vantage - FARO EUROPE GmbH \&amp; Co. KG - PDF Catalogue | Technical Documentation | Brochure." [Online]. Available: http://pdf.directindustry.com/pdf/faro-europe-gmbh-co-kg/tech-sheet-faro-laser-trackervantage/21421-258835.html. [Accessed: 05-Jun-2018].

[21] J.-F. Brethe, E. Vasselin, D. Lefebvre, and B. Dakyo, "Determination of the Repeatability of a Kuka Robot Using the Stochastic Ellipsoid Approach," in Proceedings of the 2005 IEEE International Conference on Robotics and Automation, pp. 4339-4344.

[22] F. W. Shih TY, "A Solution for Space Resection in Closed Form," 2010.

[23] E. Kalt, R. Monfared, and M. Jackson, "TOWARDS AN AUTOMATED POLISHING SYSTEM CAPTURING MANUAL POLISHING OPERATIONS," Int. J. Res. Eng. Technol., vol. 05, no. 07, pp. 182-192, 2016.

[24] G. Du and P. Zhang, "A Markerless Human-Robot Interface Using Particle Filter and Kalman Filter for Dual Robots," IEEE Trans. Ind. Electron., 2015.

[25] R. P. Paul, Robot manipulators: mathematics, programming, and control: the computer control of robot manipulators. MIT Press, 1981.

[26] "KUKA System Software 8.2 Operating \&amp; Programming Instructions pdf - CNC Manual." [Online]. Available: http://cncmanual.com/kuka-system-software-8-2-operatingprogramming-instructions/. [Accessed: 06-Jun-2018].

[27] A. Tarantola, Inverse problem theory and methods for model parameter estimation. Society for Industrial and Applied Mathematics, 2005.

[28] C. Gandrud, Reproducible Research with $R$ and $R$ Studio, Second Edition. Chapman and Hall/CRC, 2016.

[29] M. A. Noor and F. Ahmad, "Numerical comparison of iterative methods for solving nonlinear equations," Appl. Math. Comput., vol. 180, no. 1, pp. 167-172, Sep. 2006.

[30] M. Klingajay, L. D. Seneviratne, and K. Althoefer, "Identification of threaded fastening parameters using the Newton Raphson Method," Proceedings 2003 IEEE/RSJ International Conference on Intelligent Robots and Systems (IROS 2003) (Cat. No.03CH37453). IEEE.

[31] M. J. Lindstrom and D. M. Bates, "Newton-Raphson and EM Algorithms for Linear MixedEffects Models for Repeated-Measures Data," J. Am. Stat. Assoc., vol. 83, no. 404, pp. 10141022, Dec. 1988. 